\title{
Cost-effectiveness analysis of alectinib versus crizotinib in first-line treatment of anaplastic lymphoma kinase-positive advanced non-small cell lung cancer
}

Global \& Regional Health Technology

Assessment

Volume 2019: I-II

(C) The Author(s) 2019

Article reuse guidelines:

sagepub.com/journals-permissions

DOI: 10.1 | 777/22842403। 9855072

journals.sagepub.com/home/grh

@SAGE

\author{
Ravasio $\mathbf{R}^{\mathbf{I}(\mathrm{D})}$, Tiseo $\mathrm{M}^{2}$, Pradelli $\mathrm{L}^{3}$, Bellone $\mathrm{M}^{3}$, Gervasi $\mathrm{A}^{4}$ \\ and Coffani $M^{4}$
}

\begin{abstract}
In the randomized, active-controlled, multicenter Phase III open-label ALEX trial, alectinib showed superior efficacy and lower toxicity compared with crizotinib in the primary treatment of anaplastic lymphoma kinase-positive non-small cell lung cancer (ALK-positive NSCLC). The aim of this economic evaluation was to assess the cost-utility of alectinib versus crizotinib from the perspective of the Italian National Health Service (INHS). A partitioned survival model with three health states (progression-free, post-progression, and death) was used. The clinical data (progression-free survival, overall survival and time to progression) was based on the ALEX trial. Utility values were derived from EQ-5D scores evaluated in the ALEX trial and literature. Costs included drug treatments, progression-free, post-progression and supportive care. Direct medical costs and benefits (quality-adjusted life-years, QALYs) were discounted at a $3.0 \%$ annual rate. Uncertainty was assessed using deterministic and probabilistic sensitivity analyses. Treatment with alectinib versus crizotinib led to a gain of 2.82 life-years, I.86 QALYs, and incremental costs of $€ 58,276$, resulting in an incremental costutility ratio of $€ 3 \mathrm{I}, 353$ per QALY. The deterministic analysis showed that the most critical parameters in the model were the cost of post-progression and utility scores. From the probabilistic sensitivity analysis, alectinib had a $64.5 \%$ probability of being cost-effective at a willingness-to-pay threshold of $€ 40,000$ per QALY. Compared with crizotinib, alectinib increased the length of the progression-free state and the QALYs. The incremental overall cost increase was reflective of longer treatment durations in the progression-free state. Compared with crizotinib, alectinib can be considered a valid cost-utility option in the treatment of naive patients with ALK-positive NSCLC.
\end{abstract}

\section{Keywords}

Alectinib, crizotinib, NSCLC, ALK-positive, cost-utility, Italian NHS

Date received: 20 February 2019; accepted: 2 May 2019

\section{Introduzione}

In Italia, escludendo i carcinomi della cute, il carcinoma del polmone occupa il terzo posto fra i tumori a maggior frequenza di diagnosi (14\% sul totale dei tumori) ed è, rispettivamente, la prima e terza causa di morte oncologica tra maschi $(26 \%)$ e femmine $(11 \%) .{ }^{1} \mathrm{Nel} 2017$ sono stati stimati circa 41.800 nuovi casi, di cui 28.200 riferiti ai maschi e 13.600 alle femmine. ${ }^{1}$ Dato che i primi stadi del carcinoma del polmone non mostrano sintomi, o mostrano sintomi non specifici, la maggior parte dei soggetti (circa i

\author{
'Health Publishing \& Services Srl, Milano, Italia \\ ${ }^{2}$ Dipartimento Medicina e Chirurgia, Università degli Studi di Parma e \\ U.O. Oncologia Medica, Azienda Ospedaliero-Universitaria di Parma, \\ Parma, Italia \\ ${ }^{3}$ AdRes, Health Economics \& Outcome Research, Torino, Italia \\ ${ }^{4}$ Roche S.p.A., Monza, Italia
}

Corresponding author:

Roberto Ravasio, Health Publishing \& Services Srl, Piazza Duca d'Aosta 12, Milano, 20124, Italy.

Email: rravasio@aboutpharma.com 
due terzi) manifesta, al momento della diagnosi, una malattia localmente avanzata 0 metastatica. ${ }^{2} \mathrm{La}$ sopravvivenza a 5 anni negli uomini è pari al $15 \%$, mentre è lievemente più alta nelle donne $(19 \%){ }^{1}$

Il microcitoma o carcinoma polmonare a piccole cellule (small cell lung cancer, SCLC) e il carcinoma non a piccole cellule (non-small cell lung cancer, NSCLC) costituiscono oltre il $95 \%$ delle neoplasie che interessano il polmone. Di questo sottoinsieme lo SCLC costituisce circa il $15 \%$, mentre il restante $85 \%$ è ricoperto dal NSCLC. ${ }^{3}$ Quest'ultimo a sua volta è suddiviso in tre sottotipi: adenocarcinoma, carcinoma a cellule squamose e carcinoma a grandi cellule.

Il continuo progresso nella conoscenza della natura molecolare del carcinoma del polmone ha permesso lo sviluppo in questi anni di terapie a bersaglio molecolare, o targeted therapies (TT), con un conseguente miglioramento degli esiti clinici per i pazienti. ${ }^{4,5}$ La prima TT adottata nel trattamento del NSCLC è stata sviluppata per la mutazione epidermal growth factor receptor (EGFR). ${ }^{6-9}$ I pazienti trattati con un inibitore della tirosina chinasi (tyrosine kinase inhibitor, TKI) hanno mostrato, rispetto alla chemioterapia, miglioramenti sia nei tassi di risposta sia nella sopravvivenza libera da progressione (progressionfree survival, PFS) ${ }^{6-9}$ A conferma di questi risultati, le attuali linee guida internazionali raccomandano l'esecuzione del test per investigare la presenza della mutazione EGFR nei pazienti con NSCLC, nei tumori non squamosi avanzati.10,11 Successivamente, l'analisi molecolare del carcinoma del polmone ha indagato la traslocazione del gene anaplastic lymphome kinase (ALK) quale ulteriore bersaglio molecolare per una TT. Tutte le linee guida raccomandano di effettuare il test per questa mutazione su tutti i pazienti con NSCLC ad istologia non squamosa. ${ }^{12}$

Crizotinib, essendo il primo TKI mirato per il trattamento del NSCLC avanzato ALK-positivo, che ha dimostrato di essere superiore rispetto alla chemioterapia in termini di PFS, ha rappresentato fino a poco tempo fa il trattamento standard per i pazienti con NSCLC avanzato precedentemente non trattati con questa traslocazione. ${ }^{13}$ Poiché molti soggetti vanno incontro a resistenza o, raramente, non tollerano crizotinib entro il primo anno di trattamento, sono stati successivamente approvati e utilizzati altri TKIs per la gestione del NSCLC avanzato ALK-positivo. ${ }^{14-17}$ Tra questi, alectinib, un TKI di seconda generazione, ha recentemente ottenuto l'autorizzazione all'immissione in commercio dall'European Medicines Agency (EMA) nel trattamento di prima linea dei pazienti adulti con NSCLC in stadio avanzato ALK-positivo. ${ }^{18}$ Due studi clinici di Fase II ne hanno dimostrato l'efficacia e la sicurezza nei pazienti ALK-positivi precedentemente trattati, ${ }^{19,20}$ mentre lo studio clinico ALEX, internazionale, randomizzato, di Fase III ne ha dimostrato l'efficacia nel trattamento di prima linea dei pazienti con NSCLC avanzato ALK-positivo precedentemente non trattati. ${ }^{21}$ Lo studio ALEX ha confrontato alectinib rispetto a crizotinib evidenziando una maggior PFS (25,7 mesi [IC95\% 19,9 non stimabile] vs 10,4 mesi [IC95\% 7,7-14,6]) e un più basso rapport di rischio (hazard ratio, HR) per la progressione della malattia o decesso ( 0,5 IC95\% $0,36-0,70) \cdot{ }^{21}$

Alla luce della maggior efficacia di alectinib rispetto a crizotinib $^{21}$ e della crescente necessità espressa sia dai clinici sia dai decisori di stimare il valore delle nuove terapie, soprattutto in ambito oncologico, l'obiettivo di quest'analisi è stato quello di determinare, nella prospettiva del Servizio Sanitario Nazionale (SSN), il rapporto di costo efficacia (incrementale) di alectinib rispetto a crizotinib nel trattamento di una popolazione di pazienti affetti da NSCLC ALK-positivo in stadio avanzato precedentemente non trattati (pazienti naïve).

\section{Metodi}

\section{La struttura del modello}

L'analisi di costo efficacia è stata condotta utilizzando un pre-esistente modello di simulazione sviluppato in Excel (Microsoft, Redmond, WA, USA). La struttura logica di questo modello prevede tre possibili stati di salute per il paziente: i) libero da progressione (progression-free, $\mathrm{PF}$ ), ii) post progressione (post-progression, $\mathrm{PP}$ ) e iii) morte (Figura 1). Il tempo trascorso in ogni stato di salute è stato stimato applicando la metodologia indicata dalla partitioned survival analysis (PartSA). ${ }^{22}$ Con questa metodologia, un'ipotetica corte di pazienti viene seguita nel tempo attraverso una serie di stati di salute esaustivi e mutuamente esclusivi. A differenza di quanto avviene nei modelli di Markov, il numero di persone presente in qualsiasi stato di salute, a ogni istante di tempo considerato, non è determinato dalle matrici di transizione, ma stimato in base alle funzioni di sopravvivenza (libera da progressione, PFS) e complessiva (overall survival, OS) dei trattamenti oggetto del confronto. La porzione di pazienti in PP è ottenuta come differenza della sopravvivenza attesa complessiva e libera da progressione. Questo approccio è tipicamente utilizzato nei modelli di simulazione che indagano trattamenti contro il cancro dove è fondamentale poter separare le funzioni di sopravvivenza per PSF e OS.

Nel modello sono stati considerati i soli costi diretti sanitari (es. terapia oncologica, monitoraggio, postprogressione, eventi avversi, ecc.). I pazienti nello stato di salute PF ricevono il trattamento d'elezione (alectinib o crizotinib) fino a progressione. Il modello tiene conto dei costi associati alle sequenze di trattamento di seconda e terza linea per progressione del carcinoma sulla base di quanto indicato dallo studio clinico $\mathrm{ALEX}^{21}$ e dalle previsioni di mercato riferite al contesto nazionale (data on 
file). ${ }^{23} \mathrm{Al}$ fallimento di crizotinib, i pazienti possono ricevere come seconda linea di trattamento alectinib (52\%), ceritinib $(40 \%)$ o chemioterapia ( $8 \%)$, mentre dopo alectinib è stato assunto che tutti ricevano come seconda linea la sola chemioterapia. Nel modello è stata considerata come chemioterapia la somministrazione del regime pemetrexed in associazione a carboplatino. Al fallimento della seconda linea di trattamento i pazienti riceveranno, quale terza linea di trattamento, la best supportive care (BSC). La durata dei cicli del modello è stata posta uguale a una settimana in funzione dell'obiettivo di stimare con maggiore precisione la permanenza di ogni paziente in ciascun stato di salute. È stato adottato un orizzonte temporale di 30 anni (lifetime), in quanto ritenuto sufficiente per "catturare" le differenze più importanti espresse in termini sia di outcome clinico sia di costi di trattamento. ${ }^{24}$ Tale scelta è avvalorata dalla previsione di sopravvivenza stimata dal modello secondo cui a 30 anni oltre il 95\% della coorte considerata sarà deceduta. In accordo con quanto indicato dalle linee guida dell'Associazione Italiana di Economia Sanitaria (AIES), costi e benefici futuri oltre l'anno sono stati scontati applicando un tasso annuo del $3 \% .{ }^{25}$ I risultati dell'analisi di costo efficacia di alectinib vs crizotinib sono stati presentati in termini di costo incrementale per anno di vita (life-year, LY) guadagnato e di costo incrementale per anno di vita corretto per la qualità (QALY) guadagnato.

\section{Dati clinici}

I dati disponibili più recenti per valutare la PFS, la OS e la durata del trattamento (time to treatment failure, TTF) di alectinib o crizotinib sono stati ricavati dallo studio clinico ALEX. ${ }^{21}$ Le curve di PFS e OS sono state estrapolate sulla base delle rispettive curve di Kaplan-Meier, applicando specifiche distribuzioni parametriche (esponenziale, gamma, Gompertz, log-logistica, log-normale e Weibull). La scelta della distribuzione da adottare è stata effettuata sulla base di criteri multipli che includevano l'ispezione visiva, il miglior fitting ai dati (valutati con i Bayesian information criterion (BIC) e Akaike information criterion (AIC) - vedi Appendice) e la validità esterna (valutazione della plausibilità delle estrapolazioni da parte di un gruppo di oncologi specializzati nel trattamento del carcinoma polmonare). Per la PFS, il miglior fitting ai dati osservati è stato ottenuto applicando la distribuzione gamma per alectinib e quella log-normale per crizotinib (vedi Appendice). Queste distribuzioni producevano però stime di sopravvivenza ottimistiche a 10 anni dalla diagnosi, in contrasto con l'andamento clinico del carcinoma osservato nella realtà. Pertanto, seguendo l'esplicita indicazione del gruppo di oncologi, sono state adottate distribuzioni più conservative, come quella esponenziale per alectinib e quella di Weibull per crizotinib (Figura 2A). La distribuzione esponenziale e quella log-normale garantivano invece il miglior fitting per le curve di OS di alectinib e crizotinib, rispettivamente (vedi Appendice).

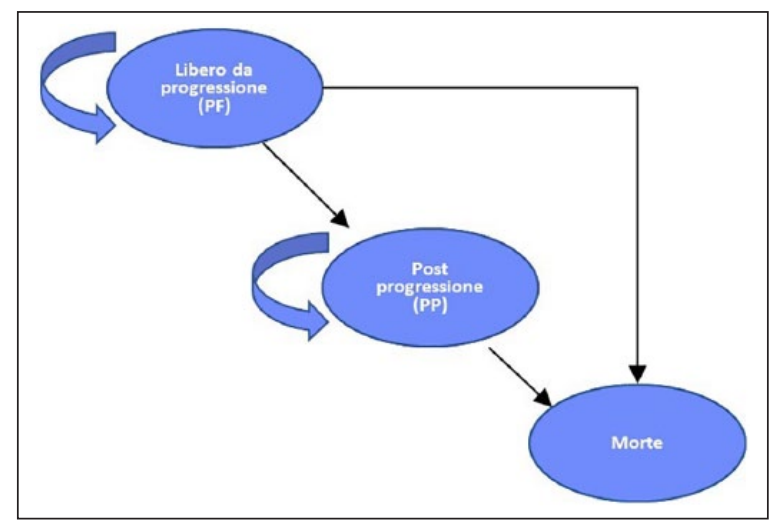

Figura I. Struttura del modello di simulazione.

Nonostante ciò, sulla base di quanto suggerito dalla validità esterna (le curve di sopravvivenza dovrebbero convergere verso un tasso di sopravvivenza medio) si è ritenuto che la distribuzione di Weibull fosse in grado di rappresentare al meglio l'estrapolazione della OS per entrambi i trattamenti (Figura 2B). Va però considerato che al momento dell'analisi il $73 \%$ pazienti arruolati in trattamento con alectinib e il $65 \%$ di quelli con crizotinib erano ancora in vita.

Infine il modello tiene conto delle progressioni dovute a metastasi cerebrali. Come per la PFS o per la OS, la percentuale di pazienti che progredisce a causa di un tumore metastatico del cervello è stata estrapolata mediante l'adozione di distribuzioni parametriche applicate alle curve di sopravvivenza libera da evento metastatico costruite sui dati dello studio ALEX. ${ }^{21}$ La distribuzione esponenziale per alectinib e quella log-normale per crizotinib presentavano il miglior fitting sui dati osservati (vedi Appendice). Le estrapolazioni così ricavate sono state validate dal gruppo di oncologi (Figura 2C). Da questi risultati si evidenzia che con crizotinib, a 14 mesi, ovvero al raggiungimento della PFS media, $1^{\prime} 80 \%$ dei pazienti manifesta metastasi cerebrali, mentre, con alectinib, al raggiungimento della PFS media - intorno ai 32 mesi - il $74 \%$ ha una progressione dovuta a lesione secondaria del cervello.

\section{Durata di trattamento}

La durata effettiva della terapia con alectinib o con crizotinib è definita come il tempo alla sospensione del trattamento (TTF) che, come indicato nello studio clinico ALEX, ${ }^{21}$ si riferisce al tempo trascorso dalla prima dose di farmaco somministrata fino alla progressione della malattia o a tossicità inaccettabile. La durata di trattamento è quindi ricavata per estrapolazione (come per la sopravvivenza libera da progressione e complessiva) applicando la distribuzione esponenziale alla curva di Kaplan-Meier del tempo alla sospensione in entrambi i trattamenti (Figura 2D - vedi 


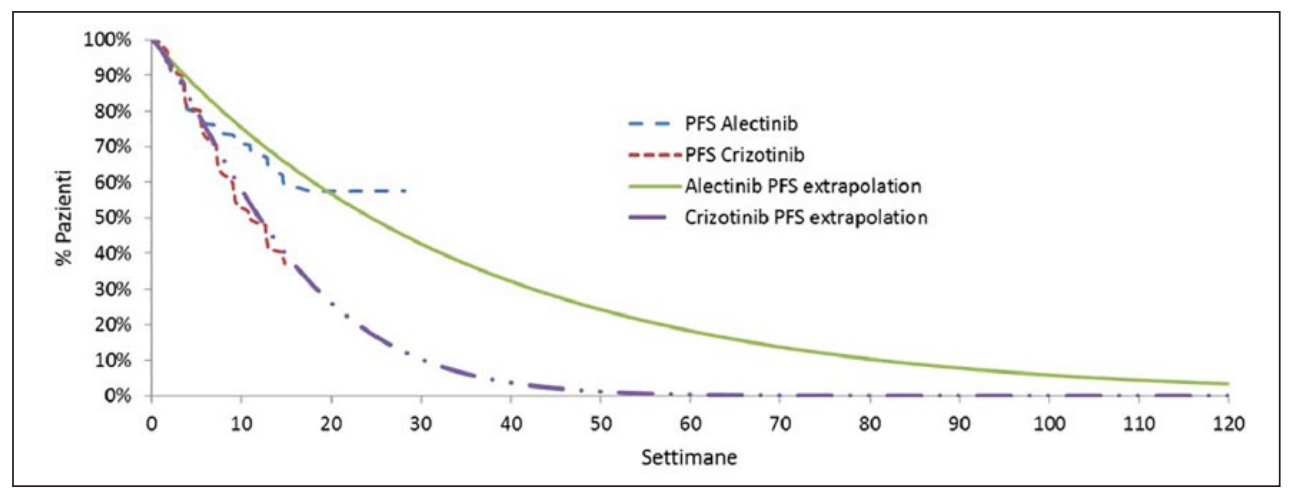

Figura 2A. Distribuzioni parametriche di sopravvivenza libero da progressione (PFS) (alectinib: esponenziale; crizotinib: Weibull) su curve di Kaplan-Meier.

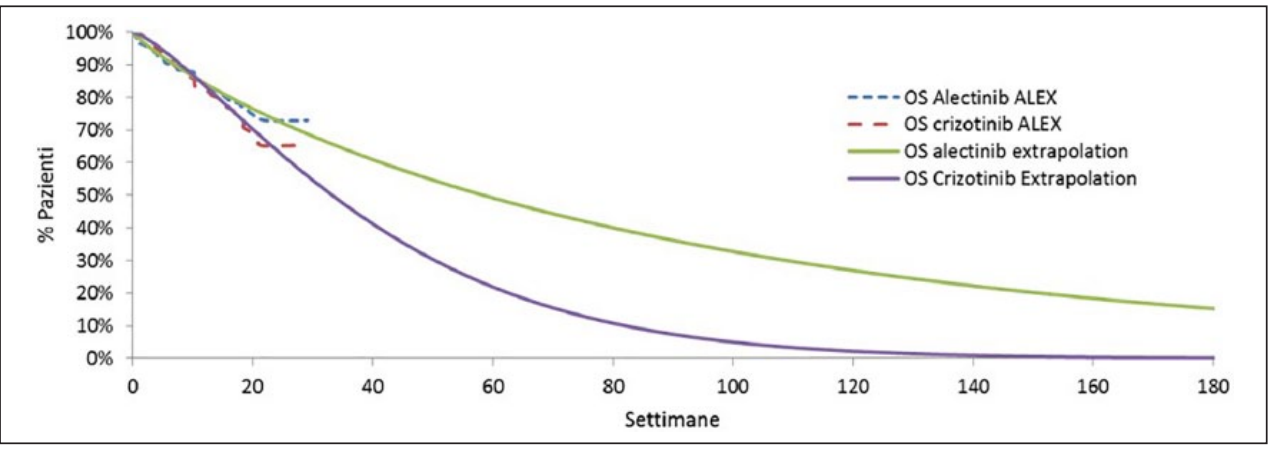

Figura 2B. Distribuzioni parametriche di sopravvivenza complessiva (OS) (alectinib: Weibull; crizotinib: Weibull) su curve di Kaplan-Meier.

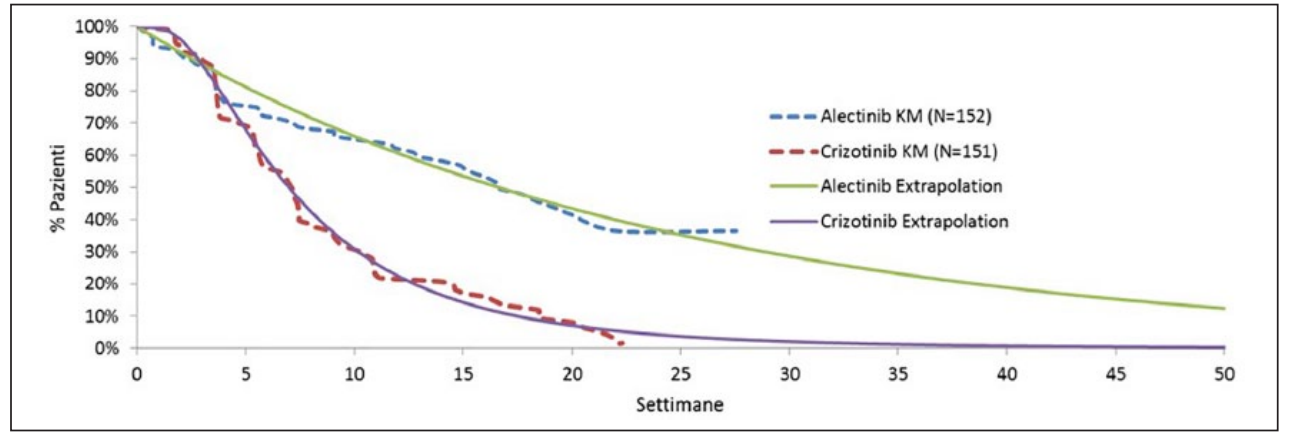

Figura 2C. Distribuzioni parametriche metastasi cerebrali (alectinib: Esponenziale; crizotinib: log-normale) su curve di KaplanMeier.

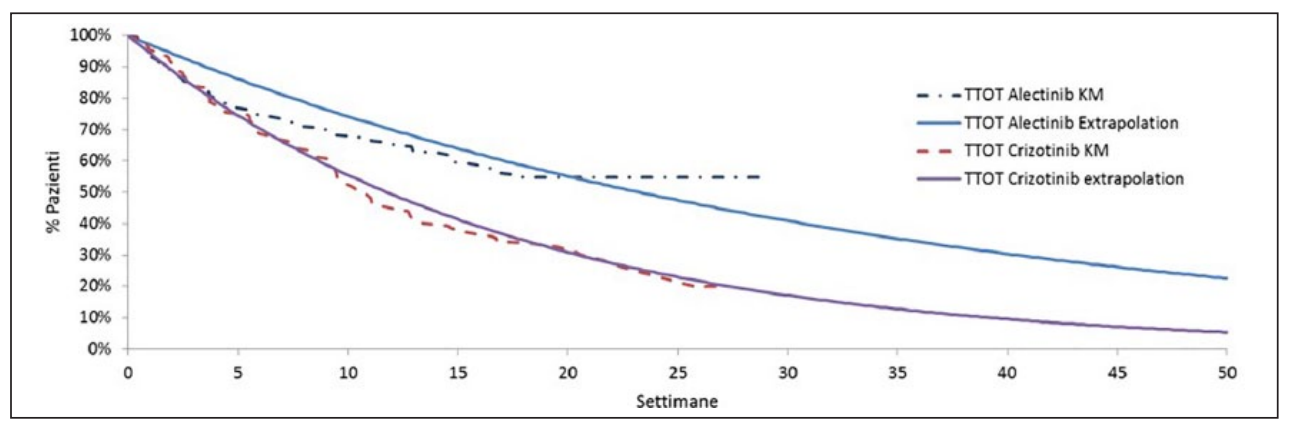

Figura 2D. Distribuzioni parametriche di tempo alla sospensione del trattamento (TTF) (alectinib: Esponenziale; crizotinib: esponenziale) su curve di Kapal-Meier. 
Appendice). Con una durata media del follow-up di 15,5 mesi per crizotinib e di 17,08 mesi per alectinib, si ricorda che per quest'ultimo nello studio ALEX al momento del follow-up non è stato ancora raggiunto il tempo mediano di TTF (IC95\%: 16,5-non stimabile [NE]), mentre per crizotinib è stato di 10,8 mesi (IC95\%: 9,4-12,9). La Tabella 1 riporta invece la durata mediana di trattamento considerata per i farmaci somministrati in seconda linea dopo fallimento delle terapie d'elezione. ${ }^{15,26}$

\section{Eventi avversi}

La Tabella 2, sulla base di quanto osservato nello studio ALEX, ${ }^{21}$ riporta, distinte per alternativa terapeutica, le probabilità associate ai principali eventi avversi di grado $3-4$, che richiedono almeno un accesso ambulatoriale e/o un ricovero ospedaliero.

Tabella I. Durata media dei trattamenti di seconda linea.

\begin{tabular}{lll}
\hline Trattamento di seconda linea & Durata (settimane) & Fonte \\
\hline Ceritinib & 42,03 & 15 \\
Alectinib & 60,41 & 26 \\
Chemioterapia & 8,86 & 26 \\
\hline
\end{tabular}

\section{Utilità}

La qualità della vita (quality of life, QoL) dei pazienti è stata valutata attribuendo specifici valori di utilità per ogni stato di salute previsto dal modello. Allo stato di PF, indipendentemente dal trattamento somministrato, è stata associata un'utilità di 0,814 (standard error $[\mathrm{SE}]$ 0,011; dato medio ottenuto per regressione dei dati a livello paziente in ALEX). Tale valore, come pure i successivi, è stato calcolato somministrando ai pazienti in trattamento con alectinib o crizotinib il questionario EQ-5D-3L durante lo studio ALEX. ${ }^{21}$ Dopo progressione (PP), come osservato nello studio ALEX, ${ }^{21}$ ai pazienti può essere somministrato un TKI ( $46 \%$ dei casi) o una chemioterapia ( $54 \%$ dei casi). Nel primo caso (TKI) è stata considerata un'utilità di 0,725 (SE 0,014), ${ }^{21}$ mentre nel secondo caso (chemioterapia) l'utilità è stata posta pari a $0,660(\mathrm{SE} 0,040) \cdot{ }^{13} \mathrm{Il}$ modello infine include anche l'utilità, posta pari a 0,470 (SE 0,101), associata ai pazienti che nello stato di PP ricevono, quale terza linea di trattamento, la best supportive care (BSC). ${ }^{27}$

\section{Costi di trattamento}

Il costo per settimana di trattamento è stato calcolato utilizzando per ogni farmaco il rispettivo schema posologico e prezzo ex-factory pubblicato in Gazzetta

Tabella 2. Incidenza degli eventi avversi di grado 3-4 per trattamento somministrato ${ }^{10}$.

\begin{tabular}{|c|c|c|c|c|c|c|}
\hline \multirow[t]{2}{*}{ Eventi avversi } & \multirow[t]{2}{*}{ Costo unitario } & \multirow[t]{2}{*}{ Fonte } & \multicolumn{2}{|c|}{ Alectinib $(n=152)$} & \multicolumn{2}{|c|}{ Crizotinib $(n=|5|)$} \\
\hline & & & $\mathrm{N}$ & $\%$ & $\mathrm{~N}$ & $\%$ \\
\hline Infezione da acinetobacter & $€ 5.983$ & 34 & 0 & $0 \%$ & I & $1 \%$ \\
\hline Anemia & $€ I .323$ & 33 & 3 & $2 \%$ & 0 & $0 \%$ \\
\hline Astenia & $€ 307$ & 33 & 1 & $1 \%$ & 0 & $0 \%$ \\
\hline Fibrillazione atriale & $€ 974$ & DRG 139 & 0 & $0 \%$ & 1 & $1 \%$ \\
\hline Arresto cardiaco & $€ I .773$ & 33 & 0 & $0 \%$ & 1 & $1 \%$ \\
\hline Diarrea & $€ 1.995$ & 33 & 0 & $0 \%$ & 3 & $2 \%$ \\
\hline Insufficienza epatica da farmaco & $€ \mathrm{I} .407$ & DRG 206 & 1 & $1 \%$ & I & $1 \%$ \\
\hline Stanchezza & $€ 307$ & 33 & 1 & $1 \%$ & 0 & $0 \%$ \\
\hline Epatotossicità generale & $€ \mathrm{I} .407$ & DRG 206 & 1 & $1 \%$ & 0 & $0 \%$ \\
\hline Coma ipoglicemico & $€ 2.489$ & DRG 23 & 1 & $1 \%$ & 0 & $0 \%$ \\
\hline Leucopenia & $€ 1.802$ & 33 & 0 & $0 \%$ & 1 & $1 \%$ \\
\hline Linfedema & $€ 728$ & DRG 284 & 0 & $0 \%$ & I & $1 \%$ \\
\hline Nausea & $€ 375$ & 33 & 0 & $0 \%$ & 3 & $2 \%$ \\
\hline Neutropenia & $€ 5 I I$ & 33 & 0 & $0 \%$ & 13 & $3 \%$ \\
\hline Edema & $€ 3.802$ & DRG 87 & 1 & $1 \%$ & 0 & $0 \%$ \\
\hline Edema periferico & $€ 3.802$ & DRG 87 & 0 & $0 \%$ & 1 & $1 \%$ \\
\hline Candidosi esofagea & $€ 959$ & DRG 183 & 0 & $0 \%$ & 1 & $1 \%$ \\
\hline Esofagite & $€ 959$ & DRG 183 & 0 & $0 \%$ & 1 & $1 \%$ \\
\hline Polmonite infettiva & $€ 5.983$ & 34 & 2 & $1 \%$ & 0 & $0 \%$ \\
\hline Polmonite $a b$ ingestis & $€ 5.983$ & 34 & 0 & $0 \%$ & 3 & $2 \%$ \\
\hline Embolia polmonare & $€ 4.009$ & DRG 78 & 0 & $0 \%$ & I & $1 \%$ \\
\hline Rash & $€ 310$ & 33 & 1 & $1 \%$ & 0 & $0 \%$ \\
\hline Insufficienza respiratoria & $€ 3.802$ & DRG 87 & 1 & $1 \%$ & 0 & $0 \%$ \\
\hline Vomito & $€ 375$ & 33 & 0 & $0 \%$ & 5 & $2 \%$ \\
\hline
\end{tabular}


Tabella 3. Costo di trattamento.

\begin{tabular}{lll}
\hline Terapia oncologica & Dosaggio die & Costo per settimana \\
\hline Alectinib & $1200 \mathrm{mg}$ & $€ I .539,52$ \\
Crizotinib & $500 \mathrm{mg}$ & $€ I .381,40$ \\
Ceritinib & $750 \mathrm{mg}$ & $€ I .420,38$ \\
Chemioterapia* & $500 \mathrm{mg} / \mathrm{m}^{2}$ & $€ 819,46$
\end{tabular}

* La dose raccomandata di pemetrexed è di $500 \mathrm{mg} / \mathrm{m}^{2}$ di superficie corporea da somministrare per infusione endovenosa in 10 minuti il primo giorno di ciascun ciclo di 21 giorni.

Ufficiale al netto delle riduzioni di legge obbligatorie (Tabella 3). Nello stato di salute PF il costo del trattamento con alectinib o crizotinib viene calcolato assumendo che i pazienti siano trattati fino alla progressione della malattia $\mathrm{o}$ fino a tossicità inaccettabile. Nello stato di salute PP il costo del trattamento farmacologico corrisponde invece a un costo medio per settimana calcolato sulla base dei possibili farmaci ipotizzati dopo la progressione e della loro durata di trattamento. Per alectinib è stata ipotizzata come seconda linea di trattamento la sola chemioterapia, mentre per crizotinib è stato ipotizzato la somministrazione di alectinib (52\% dei casi), ceritinib (40\% dei casi) o chemioterapia ( $8 \%$ dei casi). In caso di fallimento della seconda linea, i pazienti in progressione vengono associati a una terza linea di trattamento che corrisponde alla BSC con un costo per settimana di trattamento pari a $€ 140,76$. Questo valore, riferito in particolare alla terapia analgesica e nutrizionale attuata a domicilio, è stato ricavato attualizzando al 201728 i risultati di un'analisi condotta in ambito nazionale che ha avuto come obiettivo la stima dei costi e dell'efficacia delle chemioterapie usate nel trattamento di pazienti con NSCLC. ${ }^{29}$

Per alectinib, crizotinib e ceritinib non sono stati considerati costi di somministrazione in quanto farmaci orali. Mentre per la chemioterapia, oltre al costo farmacologico per l'acquisizione di pemetrexed, è stato considerato il costo associato alla somministrazione per via infusionale pari a $€ 37,10$ che corrisponde alla tariffa del DRG 410 in regime di ricovero diurno abbattuta del $90 \%$, inclusiva dei costi per carboplatino e terapia ancillare. Lo sconto sulla tariffa DRG è un provvedimento della Commissione Salute che ha approvato il Testo Unico della Mobilità Sanitaria, ratificato dalla Conferenza delle Regioni e Provincie Autonome, per adeguare il tariffario ai ricoveri finalizzati al trattamento di neoplasie con farmaci oncologici innovativi e ad alto costo, per i quali è richiesta la rendicontazione separata alla tariffa del ricovero.

L'attività di monitoraggio qui considerata riflette le raccomandazioni delle linee guida AIOM 2018. ${ }^{30}$ Per i TKI è stato ipotizzato lo stesso numero di controlli indicati dalle linee guida AIOM per chemioterapia o radioterapia. Viene suggerito un controllo clinico (visita oncologica costo unitario $€ 20,99$ cod. 89.7) dopo trattamento fino alla remissione delle tossicità acute, ogni tre-sei mesi nei primi due anni e successivamente ogni 12 mesi, due tomografie computerizzate con e senza contrasto (costo unitario $€ 124,11$ cod. 87.41.1) nei primi due anni e una nei successivi, mentre devono essere effettuate una broncoscopia (costo unitario $€ 82,36$ cod. 33.22) e una spirometria (costo unitario $€ 37,18$ cod. 89.37.2) entro l'anno dalla diagnosi. Queste attività sono state valorizzate alla luce delle relative tariffe di rimborso delle prestazioni di assistenza specialistica ambulatoriale (DM 10/2012). Il costo per settimana di trattamento è determinato assumendo che visite ed esami siano eseguite per tutta la vita, ovvero per la durata massima di 30 anni prevista dal modello.

Ai pazienti che sviluppano metastasi cerebrali è associato un costo medio forfettario di $€ 19.744$. Tale costo è stato ricavato da una precedente esperienza condotta negli Stati Uniti. ${ }^{31}$ Gli autori hanno determinato il costo incrementale dovuto alla comparsa delle metastasi cerebrali registrando retrospettivamente le risorse impiegate prima e in seguito alla diagnosi di metastasi cerebrale in una coorte di pazienti con carcinoma polmonare. Tale costo incrementale è stato convertito in Euro al 2017, sulla base degli indici purchasing power parity (PPP) (specifici per i servizi sanitari ospedalieri e per il contesto italiano. . $8,32^{2}$

Infine i costi per valorizzare gli eventi avversi sono stati determinati facendo riferimento a quanto presente in letteratura, ${ }^{33,34} \mathrm{o}$ in mancanza di informazioni, utilizzando la relativa tariffa DRG, codificata per mezzo del codice di diagnosi specifico.

\section{Analisi di sensibilità}

Per valutare la solidità del modello in risposta a scostamenti che nella realtà potrebbero verificarsi rispetto ai valori di base dei parametri utilizzati (in quanto stime campionarie), sono state condotte un'analisi deterministica (deterministic sensitivity analysis, DSA) e un'analisi probabilistica (probabilistic sensitivity analysis, PSA - Tabella 4). Nello specifico, per quanto riguarda la DSA (analisi di sensibilità a una via) sono stati fatti variare in sequenza i seguenti parametri $( \pm 20 \%$ rispetto al valore del caso base):

- utilità di alectinib nello stato $\mathrm{PF}(0,65-0,98)$,

- utilità di crizotinib nello stato $\mathrm{PF}(0,65-0,98)$,

- utilità nello stato di $\mathrm{PP}(0,58-0,87)$,

- costo PF nei pazienti trattati con alectinib $(€ 2,57-€ 3,84)$,

- costo PF nei pazienti trattati con crizotinib $(€ 2,57-€ 3,84)$,

- costo PP nei pazienti trattati con alectinib $(€ 141,69-€ 211,51)$

- costo PP nei pazienti trattati con crizotinib (€634,29-€946,85)

- costo per la gestione delle metastasi cerebrali $(€ 15.795-€ 23.692)$ 
Tabella 4. Parametri e dettagli per l'analisi di sensibilità probabilistica.

\begin{tabular}{|c|c|c|c|c|}
\hline & Media & Range (MIN-MAX) & Standard Error & Distribuzione usata \\
\hline PFS alectinib: parametri distribuzione esponenziale & $\operatorname{lnt}=3,5635$ & - & SE (int) $=0,1270$ & Normale \\
\hline PFS crizotinib: parametri distribuzione Weibull & $\begin{array}{l}\text { Int }=2,7682 \\
\text { Scale }=0,77 \mid 5\end{array}$ & - & $\begin{array}{l}\text { SE }(\text { Int })=0,0766 \\
\text { SE }(\text { scale })=0,0642 \\
\text { Covariata }=0,0003\end{array}$ & Normale bivariata \\
\hline OS alectinib: parametri distribuzione Weibull & $\begin{array}{l}\text { Int }=4,4817 \\
\text { Scale }=1,1286\end{array}$ & - & $\begin{array}{l}\text { SE }(\text { Int })=0,3110 \\
\text { SE }(\text { scale })=0,1800 \\
\text { Covariata }=0,0442\end{array}$ & Normale bivariata \\
\hline OS crizotinib: parametri distribuzione Weibull & $\begin{array}{l}\text { Int }=3,7799 \\
\text { Scale }=0,7529\end{array}$ & - & $\begin{array}{l}\text { SE }(\text { Int })=0,1713 \\
\text { SE }(\text { scale })=0,1078 \\
\text { Covariata }=0,0133\end{array}$ & Normale bivariata \\
\hline TTF alectinib: parametri distribuzione esponenziale & $\ln t=3,5131$ & - & $S E(\ln t)=0,1213$ & Normale \\
\hline TTF crizotinib: parametri distribuzione esponenziale & $\ln t=2,8333$ & - & $S E(\ln t)=0,0976$ & Normale \\
\hline Valori di utilità in PFS & $0,8 \mid 4$ & - & 0,012 & Beta \\
\hline Valore di utilità in PP ALK inibitori & 0,725 & - & 0,014 & Beta \\
\hline Valori di utilità in PP non ALK inibitori & 0,660 & - & 0,040 & Beta \\
\hline Valori di utilità BSC & 0,470 & - & 0,101 & Beta \\
\hline Costi sopravvivenza libera da progressione & $€ 3,20$ & $€ 2,57-3,84^{*}$ & $0,10 * *$ & Log-normale \\
\hline Costi sopravvivenza dopo progressione - alectinib & $€ 176,60$ & $€|4|, 69-21|, 5|^{*}$ & $0,10 * *$ & Log-normale \\
\hline Costi sopravvivenza dopo progressione - crizotinib & $€ 790,57$ & $€ 634,29-946,85^{*}$ & $0,10 * *$ & Log-normale \\
\hline
\end{tabular}

PFS: progression-free survival; OS: overall survival; TTF: time to treatment failure; PP: post-progression; ALK: anaplastic lymphoma kinase; BSC: best supportive care; Int: intercetta; SE: standard error.

*range $\pm 20 \%$ del valore medio (assunzione); $* *$ SE calcolato come $(\log (\max )-\log (\min )) / 4$.

Per quanto riguarda invece l'analisi probabilistica, sono state effettuate 1000 iterazioni del modello, facendo ogni volta variare simultaneamente e casualmente i valori dei parametri secondo le distribuzioni probabilistiche loro assegnate e ricalcolando i risultati. Di seguito vengono presentati i parametri sui quali è stata condotta la PSA:

- parametri delle funzioni parametriche di PFS, OS, e TTF

- valori di utilità

- costi associati alla sopravvivenza libera da progressione

- costi associati alla sopravvivenza dopo progressione

Il prodotto della PSA è stato quindi presentato nelle forme del piano di costo-efficacia (cost-effectiveness plane) e della curva di accettabilità (cost-effectiveness acceptability curve). Il piano di costo-efficacia mostra la dispersione dei mille risultati delle iterazioni, ciascuno in funzione delle rispettive variazioni incrementali di beneficio (QALY) e di costo di alectinib rispetto a crizotinib. La curva di accettabilità, sempre sulla base delle 1000 iterazioni, indica (percentualmente) le frequenze con cui l'incremental cost-effectiveness ratio (ICER), il rapporto tra la variazione incrementale del costo e la variazione incrementale del beneficio di alectinib rispetto a crizotinib) risulta inferiore a un determinato valore di soglia; ovvero fornisce la probabilità che, per un determinato valore di soglia, il trattamento sia costo efficace.

\section{Risultati}

Il tempo medio trascorso nello stato di salute libero da progressione associato alla somministrazione della prima linea di trattamento con alectinib è stimato dal modello in 2,74 anni, mentre la sopravvivenza media in 5,97 anni (Tabella 5). In entrambi i casi, rispetto alla prima linea con crizotinib, si registra un incremento della durata media della PF e della OS di 1,54 anni e 2,82 anni, rispettivamente (Tabella 5). Dopo aver corretto il tempo medio di PF e di OS per la qualità della vita, i rispettivi QALY di alectinib determinano un incremento di 1,23 e di 1,86 rispetto a quelli stimati per crizotinib (Tabella 5).

Il maggior tempo trascorso nello stato di PF dal paziente trattato con alectinib determina dei costi più alti, relativamente a tale stato, rispetto al paziente trattato con crizotinib (€211.288 vs €100.055) (Tabella 5). Differentemente, per crizotinib sono maggiori i costi associati allo stato PP alla luce di una maggiore permanenza (in tale stato di salute) rispetto ad alectinib (€80.413 vs €31.838) (Tabella 5). Il costo per la gestione delle metastasi cerebrali è di $€ 8.369$ per alectinib e di $€ 12.751$ per crizotinib. In totale il paziente trattato con alectinib riporta un incremento dei costi per $€ 58.276$ ( $€ 251.495$ vs $€ 193.219)$ (Tabella 5). Ciò determina un costo incrementale per anno di vita guadagnato di $€ 20.683$ e un costo incrementale per QALY guadagnato di $€ 31.353$ (Tabella 5). Se si confronta infine il valore di questo secondo ICER col valore di soglia di $€ 40.000$ per QALY indicato dall'AIES, ${ }^{25}$ alectinib si può ritenere un'opzione terapeutica costo-efficace. 
Tabella 5. Risultati dell'analisi di costo efficacia.

\begin{tabular}{|c|c|c|c|}
\hline & Alectinib & Crizotinib & Alectinib vs Crizotinib \\
\hline LY & 5,97 & 3,16 & 2,82 \\
\hline - in PF & 2,74 & 1,21 & 1,54 \\
\hline - in progressione & 3,23 & 1,95 & 1,28 \\
\hline QALY & 3,76 & 1,90 & I,86 \\
\hline- in $P F$ & 2,20 & 0,98 & 1,23 \\
\hline - in progressione & 1,55 & 0,92 & 0,63 \\
\hline Costi in PF & $€ 211.288$ & $€ 100.055$ & $€ । I I .234$ \\
\hline - farmaci & $€ 210.417$ & $€ 99.327$ & $€ I I I .090$ \\
\hline - gestione eventi avversi & $€ 4 / 2$ & $€ 526$ & $-€ I / 3$ \\
\hline$-B S C$ & $€ 459$ & $€ 202$ & $€ 257$ \\
\hline Costi in PP & $€ 31.838$ & $€ 80.413$ & $-€ 48.575$ \\
\hline Costi metastasi cerebrali & $€ 8.369$ & $€ \mid 2.75 I$ & $-€ 4.382$ \\
\hline Costo per paziente & $€ 251.495$ & $€ 193.219$ & $€ \mathbf{5 8 . 2 7 6}$ \\
\hline ICER per LY & $€ 20.683$ & & \\
\hline ICER per QALY & $€ 31.353$ & & \\
\hline
\end{tabular}

LY: life-years; PF: progression-free; QALY: quality-adjusted life-years; BSC: best supportive care; PP: post-progression; ICER: incremental costeffectiveness ratio.

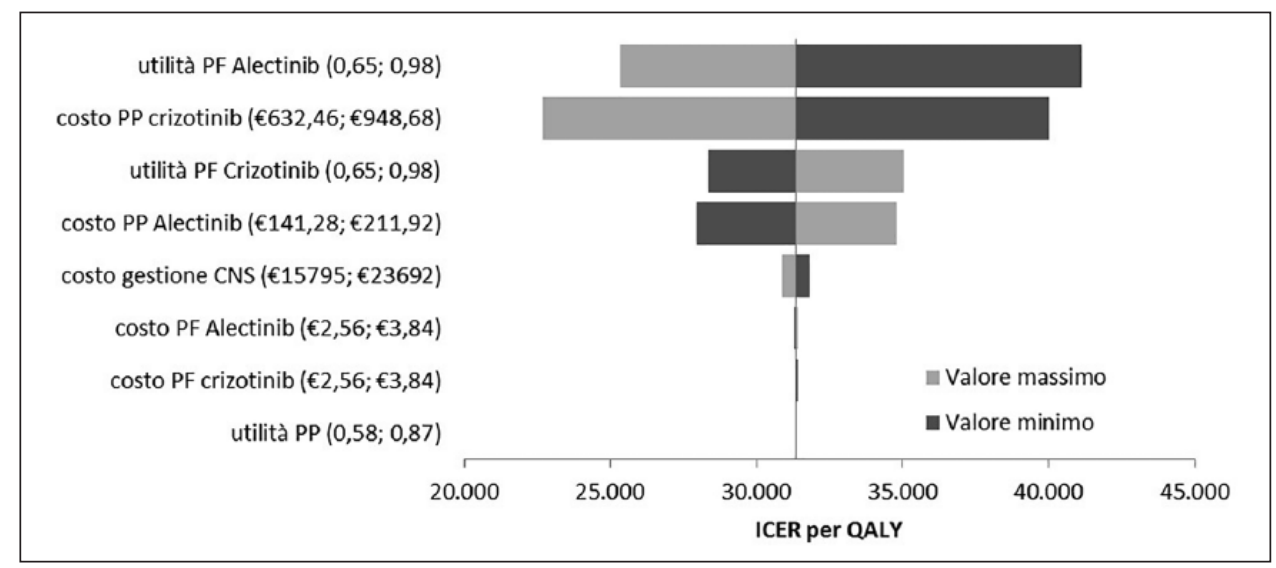

Figura 3. Diagramma a tornado: analisi di sensibilità deterministica.

D'ora in avanti nel testo, salvo diversamente specificato, si intenderà che nell'ICER il guadagno di vita è espresso in QALY.

\section{Analisi di sensibilità}

I risultati dell'analisi di sensibilità deterministica mostrano come l'utilità di alectinib e crizotinib nello stato di PF e il costo dello stato di PP per i pazienti trattati con alectinib o con crizotinib costituiscano i principali driver dell'analisi di costo efficacia incrementale (Figura 3). Il diagramma a tornado mostra il range di impatto determinato dalle principali variabili sul rapporto incrementale di costo efficacia di alectinib rispetto a crizotinib (Figura 3 ).

Per quanto riguarda invece l'analisi probabilistica, il piano di costo-efficacia mostra come tutta la dispersione delle 1000 iterazioni sia racchiusa nel primo quadrante; ciò significa che alectinib sarebbe sempre, in tutti i casi, più efficace e più costoso di crizotinib (Figura 4). Il grosso punto al centro della nuvola rappresenta l'incrocio tra QALY e costo incrementale riferiti al caso base (Figura 4). Sempre sulla base delle 1000 iterazioni, la Figura 5 (curva di accettabilità) indica che la probabilità che l'ICER di alectinib rispetto a crizotinib sia inferiore a un valore soglia di $€ 40.000^{25}$ sarebbe pari al 64,5\%.

\section{Discussione e conclusioni}

La disponibilità dei TKI ha determinato notevoli progressi nel trattamento del carcinoma polmonare non a piccole cellule, così come ha sollevato, allo stesso tempo, interesse da parte degli Enti Regolatori circa i costi di tali farmaci, da cui la necessità di valutarne la costo-efficacia. Proprio in riferimento a quest'aspetto, la necessità di condurre 


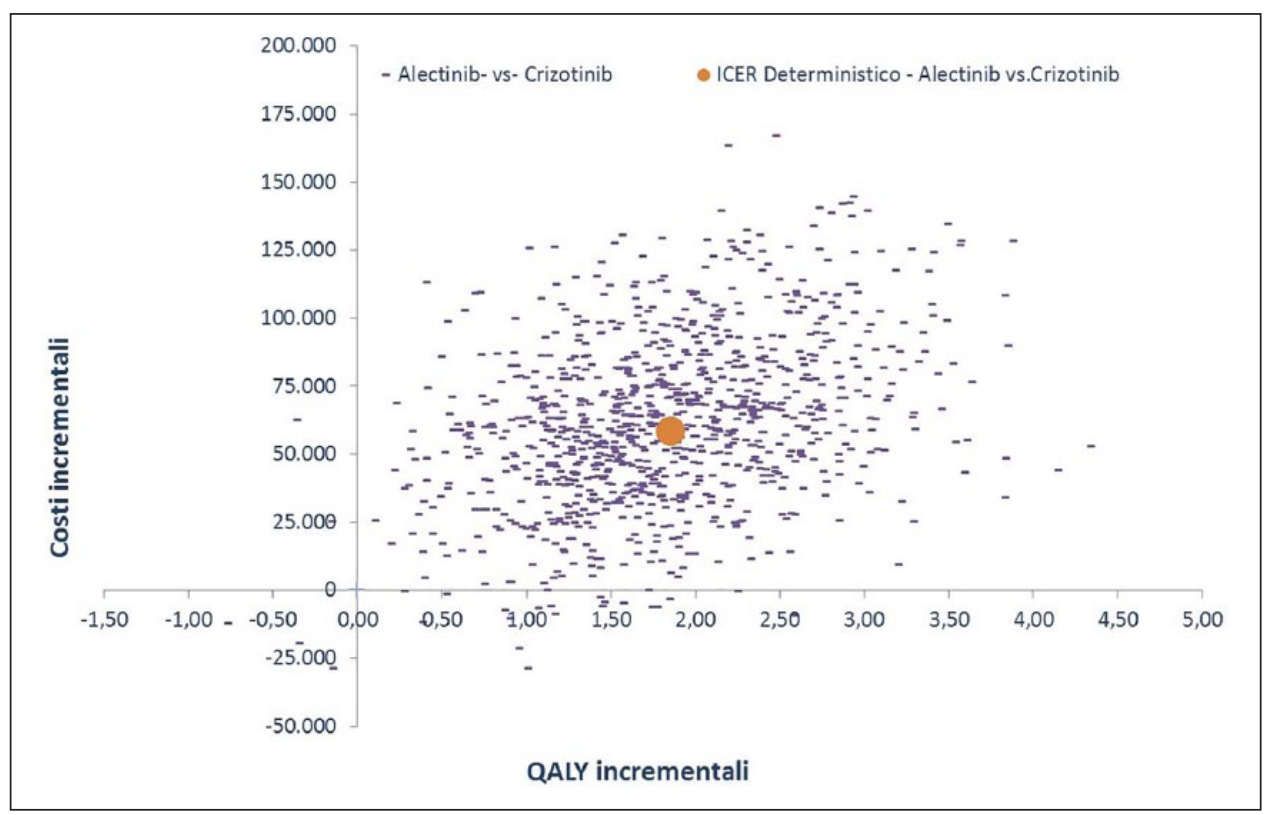

Figura 4. Piano di costo efficacia.

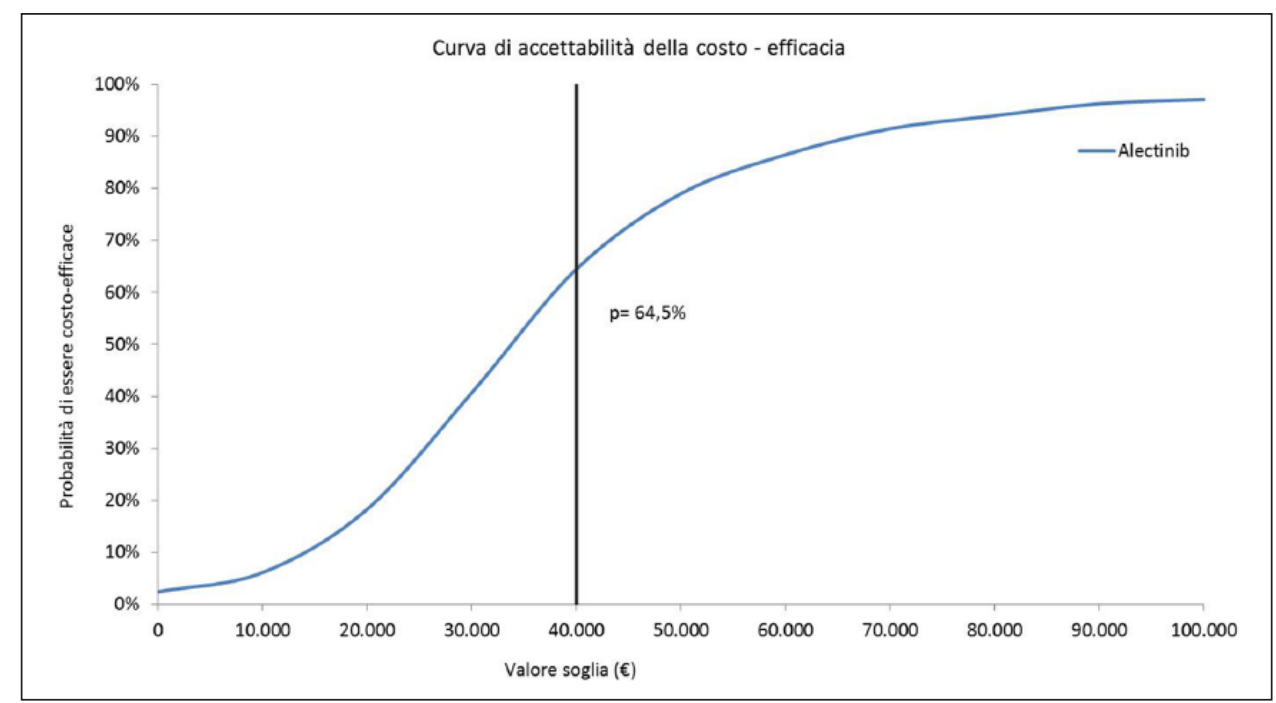

Figura 5. Curva di accettabilità di alectinib.

analisi economiche lungo un periodo lifetime, si scontra con la relativamente breve durata dei trial. Si giustifica così il ricorso a modelli di simulazione in grado di estrapolare sul lungo periodo gli esiti clinici dei trial stessi. Seguendo questa logica, il presente modello di simulazione, attraverso una semplice e trasparente rappresentazione del percorso clinico del paziente con NSCLC ALK positivo in stadio avanzato, ha permesso di stimare, lungo un periodo lifetime, il rapporto di costo efficacia di alectinib rispetto a crizotinib nel trattamento di prima linea.

L'analisi, basata sull'utilizzo di un partitioned survival model popolato principalmente con i dati clinici dello studio ALEX, ${ }^{21}$ ha riportato, nella prospettiva del SSN italiano, un costo medio incrementale di alectinib rispetto a crizotinib per LY guadagnato di $€ 20.683$ e per QALY guadagnato di $€ 31.353$. I maggiori costi del trattamento farmacologico associati ad alectinib vengono in parte controbilanciati dalla riduzione dei costi per la gestione dello stato di salute PP e delle metastasi cerebrali. Rispetto a crizotinib, infatti, la maggior efficacia di alectinib, in termini di prevenzione e remissione dei tumori secondari dell'encefalo, determina un risparmio di risorse per la gestione degli eventi metastatici correlati. È infine importante sottolineare come un costo incrementale per 
QALY inferiore a $€ 40.000$ sia considerato accettabile nella letteratura nazionale. ${ }^{25}$

Tuttavia i risultati della presente analisi rimangono legati a zone d'incertezza, come ad esempio la sopravvivenza complessiva (OS). Si è visto infatti che, non disponendo di osservazioni abbastanza prolungate nel tempo, le OS di alectinib e di crizotinib sono state costruite estrapolando mediante specifiche distribuzioni parametriche le rispettive curve di Kaplan-Meier calcolate nello studio ALEX. ${ }^{21}$

In generale, la scelta delle distribuzioni parametriche da utilizzare per l'estrapolazione della OS per le due alternative terapeutiche comporta spesso una certa aleatorietà. A tal proposito si ricorda che, seguendo l'esplicita indicazione del gruppo di oncologi, sono state adottate le distribuzioni più conservative per i due TKI oggetto del confronto rispetto all'utilizzo di distribuzioni con migliori valori di fit che però producevano stime di sopravvivenza ottimistiche a 10 anni dalla diagnosi, in contrasto con l'andamento clinico del carcinoma osservato nella realtà.

Una seconda osservazione riguarda la stima dei costi di trattamento farmacologici post progressione, poiché i diversi trattamenti assegnati ai pazienti in seguito al fallimento della prima linea (post progressione) determinano un grande impatto sul costo complessivo. Un riscontro delle ipotesi qui adottate con futuri ed esaustivi dati di consumo dei farmaci utilizzati in seconda linea sarà di primaria importanza per la validità dei risultati qui forniti.

$\mathrm{Si}$ è quindi proceduto a indagare l'incertezza sui parametri chiave del modello mediante l'analisi di sensibilità che, nel complesso, ha confermato la solidità dei risultati del caso base. In particolare la curva di accettabilità della costo-efficacia per QALY, derivata dall'analisi probabilistica (PSA), ha evidenziato una probabilità del $64,5 \%$ che alectinib sia costo-efficace rispetto a crizotinib, a fronte di una soglia di accettabilità posta pari a $€ 40.000 .{ }^{25}$ Considerando invece una soglia di accettabilità di $€ 60.000,{ }^{35}$ la probabilità che alectinib sia costo-efficace rispetto a crizotinib salirebbe allo $86,5 \%$.

I presenti risultati vengono inoltre confermati da quelli di un recente studio internazionale condotto negli Stati Uniti. ${ }^{36}$ Lo studio americano ha stimato, anch'esso lungo un orizzonte temporale lifetime, l'efficacia e i costi di trattamento di alectinib e crizotinib nel trattamento di prima linea del NSCLC ALK-positivo avanzato. Gli Autori hanno utilizzato un partitioned survival model simile a quello usato in questa sede (tre stati di salute: PF, PP e decesso). Alectinib ha determinato, rispetto a crizotinib, un ICER per LY di US\$37.611 e un ICER per QALY di US\$39.312.

Si può pertanto concludere che alectinib rappresenta una valida aggiunta alle opzioni di trattamento disponibili per i pazienti con NSCLC ALK-positivo avanzato. A conferma dei risultati presentati in questa sede si riporta anche il recente aggiornamento dei dati di PFS relativo all'estensione del periodo di follow-up dello studio ALEX $^{37}$ : alectinib ha evidenziato un incremento nella differenza di efficacia misurata in termini di PFS nei confronti di crizotinib $(38,4$ mesi vs 10,9 mesi) rispetto a quanto riportato nella prima analisi $(25,7$ mesi vs 10,4 mesi). ${ }^{21}$

\section{Declaration of Conflicting Interest}

R.R., T.M., P.L., and B.M. declare no conflict of interest. G.A and C.M. are full-time employees of Roche S.p.A.

\section{Funding}

This research was made possible by an educational grant from Roche S.p.A.

\section{Supplemental material}

Supplemental material for this article is available online.

\section{ORCID iD}

Ravasio R (iD https://orcid.org/0000-0002-5477-1957

\section{Bibliografia}

1. AIOM, AIRTUM e Fondazione AIOM (a cura di). I numeri del cancro in Italia 2018. https://www.aiom.it/wp-content/ uploads/2018/10/2018 NumeriCancro-operatori.pdf (Ultima data di accesso $2 \overline{8}$ maggio 2018).

2. Lim C, Sung M, Shepherd FA, et al. Patients with Advanced Non-Small Cell Lung Cancer: Are Research Biopsies a Barrier to Participation in Clinical Trials? J Thorac Oncol 2016; 11(1): 79-84.

3. Travis WD, Brambilla E, Nicholson AG, et al. The 2015 World Health Organization Classification of Lung Tumors: Impact of Genetic, Clinical and Radiologic Advances Since the 2004 Classification. J Thorac Oncol 2015;10(9): 12431260.

4. Reck M and Paz-Ares L. Immunologic checkpoint blockade in lung cancer. Semin Oncol 2015; 42: 402-417.

5. Gridelli C, de Marinis F, Cappuzzo F, et al. Treatment of advanced non-small-cell lung cancer with epidermal growth factor receptor (EGFR) mutation or ALK gene rearrangement: results of an international expert panel meeting of the Italian Association of Thoracic Oncology. Clin Lung Cancer 2014; 15: 173-181.

6. Mok TS, Wu YL, Thongprasert S, et al. Gefitinib or carboplatin-paclitaxel in pulmonary adenocarinoma. $N$ Engl J Med 2009; 361: 947-957.

7. Rosell R, Carcereny E, Gervais R, et al. Erlotinib versus standard chemotherapy as first-line treatment for European patients with advanced EGFR mutation-positive nonsmallcell lung cancer (EURTAC): a multicentre, open-label, randomised phase 3 trial. Lancet Oncol 2012; 13: 239-46.

8. Sequist LV, Yang JC, Yamamoto $\mathrm{N}$ et al. Phase III study of afatinib or cisplatin plus pemetrexed in patients with metastatic lung adenocarcinoma with EGFR mutations. J Clin Oncol 2013; 31(27): 3327-34. 
9. Planchard D, Popat S, Kerr K, et al. Metastatic non-small cell lung cancer: ESMO Clinical Practice Guidelines for diagnosis, treatment and follow-up. Ann Oncol 2018; 29 (Supplement_4): iv192-iv237.

10. Leighl NB, Rekhtman N, Biermann WA, et al. Molecular testing for selection of patients with lung cancer for epidermal growth factor receptor and anaplastic lymphoma kinase tyrosine kinase inhibitors: American Society of Clinical Oncology endorsement of the College of American Pathologists/International Association for the study of lung cancer/association for molecular pathology guideline. J Clin Oncol 2014; 32: 3673-3679.

11. National Comprehensive Cancer Network. NCCN clinical practice guidelines in oncology: Non-small cell lung cancer. Fort Washington: NCCN, Inc., 2017.

12. Lindeman NI, Cagle PT, Beasly MB, et al. Molecular testing guideline for selection of lung cancer patients with EGFR and ALK tyrosine kinase inhibitors. J Thorac Oncol 2013; 8(7): 823-859.

13. Solomon B, Mok T, Kim D, et al. First-line crizotinib versus chemotherapy in ALK-positive lung cancer. $N$ Engl $J$ Med 2014; 371(23): 2167-2177.

14. Doebele RC, Pilling AB, Aisner DL, et al. Mechanisms of resistance to crizotinib in patients with ALK gene rearranged non-small cell lung cancer. Clin Cancer Res 2012; 18(5): 1472-1482.

15. Shaw AT, Kim TM, Crinò L et al. Ceritinib versus chemotherapy in patients with ALK-rearranged non-smallcell lung cancer previously given chemotherapy and crizotinib (ASCEND-5): a randomised, controlled, open-label, phase 3 trial. Lancet Oncol 2017; 18(7): 874-886.

16. Novello S, Mazières $\mathrm{J}$, Oh IJ et al. Alectinib versus chemotherapy in crizotinib-pretreated anaplastic lymphoma kinase (ALK)-positive non-small-cell lung cancer: results from the phase III ALUR study. Ann Oncol 2018; 29(6): 1409-1416.

17. Kim DW, Tiseo M, Ahn MJ, et al. Brigatinib in Patients With Crizotinib-Refractory Anaplastic Lymphoma KinasePositive Non-Small-Cell Lung Cancer: A Randomized, Multicenter Phase II Trial. J Clin Oncol 2017; 35 (22): 2490-2498.

18. European Medicines Agency. Alecensa (alectinib): European Public Assessment Report. https://www.ema.europa.eu/en/ documents/overview/alecensa-epar-summary-public_it.pdf (Ultima data di accesso 28 maggio 2019).

19. Ou SI, Ahn JS, De Petris L, et al. Alectinib in crizotinibrefractory ALK-rearranged non-small-cell lung cancer: a phase II global study. J Clin Oncol 2016; 34: 661-668.

20. Seto T, Kiura K, Nishio M, et al. CH5424802 (RO5424802) for patients with ALK-rearranged advanced non-small-cell lung cancer (AF-001JP study): a single-arm, open-label, phase 1-2 study. Lancet Oncol 2013; 14(7): 590-598.

21. Peters S, Camidge DR, Shaw AT, et al. Alectinib versus crizotinib in untreated ALK-positive nonsmallcell lung cancer. N Engl J Med 2017; 377(9): 829-838.

22. Partitioned Survival Model. York: York Health Economics Consortium, 2016. https://www.yhec.co.uk/glossary/ partitioned-survival-model/ (Ultima data di accesso 28 maggio 2019).
23. Data on file, Roche.

24. Caro JJ, Briggs AH, Siebert U, et al. Modeling good research practices - overview: A report of the ISPORSMDM modeling good research practices task force-1. Value Health 2012; 15: 796-803.

25. Gruppo di lavoro AIES (Associazione Italiana di Economia Sanitaria) (coordinato da G. Fattore). Proposta di linee guida per la valutazione economica degli interventi sanitari in Italia. Pharmacoeconomics Ital Res Articles 2009; 11(2): 83-93.

26. Wolf J, Oh I, -JMazieres J, et al. ALUR: a phase 3 study of alectinib versus chemotherapy in previously treated ALK+ non-small cell lung cancer (NSCLC). Ann Oncol 2016; 27(Issue suppl_6): 1290TiP.

27. Nafees B, Stafford M, Gavriel S, et al. Health state utilities for non small cell lung cancer. Health Qual Life Outcomes 2008; 6: 84 .

28. Eurostata. Harmonised Indices of Consumer Prices (HICPs). https://ec.europa.eu/eurostat/tgm/table.do?tab=table\&init= $1 \&$ language $=$ en $\&$ pcode $=$ tec $00027 \&$ plugin $=1 \quad($ Ultima data di accesso 28 maggio 2019).

29. M Eandi. Analisi costo-efficacia della duplice chemioterapia paclitaxel + carboplatino vs combinazioni alternative nel trattamento del tumore polmonare non a piccole cellule. Farmeconomia e percorsi terapeutici 2006; 7(2): 97-117.

30. Associazione Italiana di Oncologia Medica (AIOM). Linee guida AIOM. Neoplasie del polmone. Edizione 2018. https:// www.aiom.it/wp-content/uploads/2018/11/2018_LG_AIOM_ Polmone.pdf (Ultima data di accesso 28 maggio 2019 ).

31. Guerin A, Sesane M, Dea K, et al. The economic burden of brain metastasis among lung cancer patients in the United States. J Med Econ 2016; 19(5): 526-536.

32. Organization for Economic Co-operation and Development (OECD). 2014 Purchasing Power Parities Benchmark results. https://stats.oecd.org/Index.aspx?DataSetCode=PPP2014 (Ultima data di accesso 28 maggio 2019).

33. Mickisch G, Gore M, Escudier B, et al. Costs of managing adverse events in the treatment of first-line metastatic renal cell carcinoma: bevacizumab in combination with interferon-alpha2a compared with sunitinib. $\mathrm{Br} J$ Cancer 2010; 102(1): 80-86.

34. Brown B, Diamantopoulos A, Bernier J, et al. An economic evaluation of cetuximab combined with radiotherapy for patients with locally advanced head and neck cancer in Belgium, France, Italy, Switzerland, and the United Kingdom. Value Health 2008; 11(5):791-799.

35. Messori A, Santarlasci B, Trippoli S, et al. Controvalore economico del farmaco e beneficio clinico: stato dell'arte della metodologia ed applicazione di un algoritmo farmacoeconomico. Pharmacoeconomics Ital Res Articles 2003; 5: 3-17.

36. Carlson JJ, Suh K, Orfanos P, et al. Cost Effectiveness of Alectinib vs. Crizotinib in First-Line Anaplastic Lymphoma Kinase-Positive Advanced Non-Small-Cell Lung Cancer. Pharmacoeconomics 2018; 36(4): 495-504.

37. Camidge DR, Peters S, Tony Mok T, et al. Updated efficacy and safety data from the global phase III ALEX study of alectinib (ALC) vs crizotinib (CZ) in untreated advanced ALK+ NSCLC. J Clin Oncol 2018; 36: 15 _suppl: 9043-9043. 